\title{
A Traffic Chaos Reduction Approach for Emergency Scenarios
}

\author{
Syed Rashid Ali Rizvi ${ }^{\psi}$, Stephan Olariu ${ }^{\psi}$, Mona E. Rizvi ${ }^{\mathrm{T}}$, Michele C. Weigle ${ }^{\psi}$ \\ Old Dominion University, Norfolk, VA 23529\%, Norfolk State University, Norfolk, VA $23504^{T}$ \\ (srizvi,olariu,mweigle)@cs.odu.edu,mrizvi@nsu.edu
}

\begin{abstract}
This paper proposes an efficient chaos-reducing information dissemination approach for spatiotemporal traffic information related to first responders and planned evacuation scenarios using Vehicular Ad Hoc Networks (VANETs). VANETs have recently been proposed as one of the promising ad-hoc networking techniques that can be used to provide a safe and enjoyable driving experience. In our approach, we provide an emergency vehicle path clearing technique, and real-time resource (e.g. shelter) availability information. Therefore, traffic confusion and chaos is lowered on evacuation and emergency vehicle routes. Simulation results show that our approach works efficiently without fully relying on any message relaying infrastructure.
\end{abstract}

\section{Introduction}

One of the major causes of traffic chaos is the confusion and failure to detect Emergency Service Vehicles (ESVs) (e.g. ambulances, police cars and fire trucks, etc.), which can lead to slow progress and even accidents involving the ESVs traveling towards their destinations. In addition to ESV scenarios, during an evacuation, there exists a tremendously challenging task for supply trucks to reach their destinations as quickly as possible and with minimal interruptions (see Figure 1).

Vehicular Ad Hoc Networks (VANETs) have recently been proposed as a technology that can provide both drivers and passengers with a safe and enjoyable driving experience. Although research has been done on evacuation using infrastructure-based Intelligent Transportation Systems (ITS) [1], less attention has been given to proposing a solution with a combination of VANET and evacuation rescue efforts. In this paper, we propose a VANET-based approach that reduces the chaos and confusion among evacuees and first responders. ITS technologies can be used to gather traffic, weather, and other information; however, the concentration of ITS deployment is in urban areas, and evacuation travel mostly occurs in rural areas [1]. Since we do not fully rely on any message relaying infrastructure, our solution is suited for rural areas.

\section{VANET system model}

In our approach, all vehicles act as information servers relaying information for the VANET. We do not make use of any access points on the road side or depend upon a collection of location servers. We assume that every vehicle has a digital map and knows its geographical position and heading through a Global Positioning System (GPS) receiver. The building blocks of our approach are:

- Resources: ESVs, parking places, gas stations, hospitals, shelters, etc;

- Reports: Information sent by resources;

- Broadcasting: We exploit broadcasting for information propagation and take the spatiotemporal character of reports into account;

- Selection strategies based on relevance: The spatial distribution of information is controlled through a selection strategy.

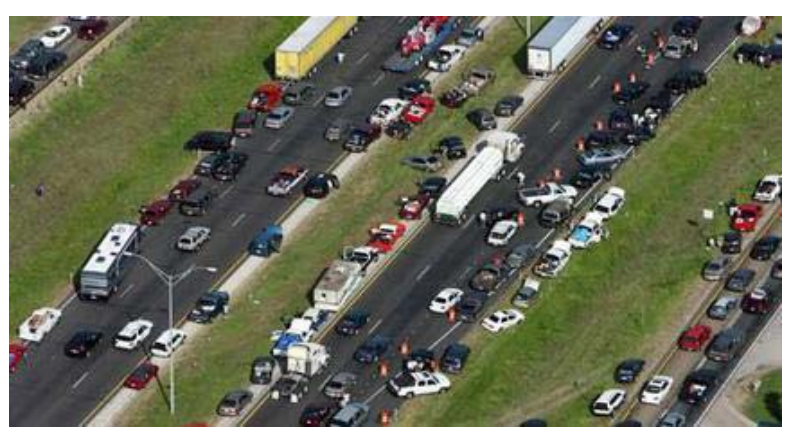

Figure 1. Cars sit broken down or without fuel by the side of the road in Huntsville, Texas. Trucks filled with life-saving relief supplies are unable to reach their destinations [2]. 


\section{Traffic Chaos Reduction Approaches}

\subsection{ESV route traffic chaos reduction approach}

When an emergency service is called, one or more ESVs can be dispatched to a destination. Since the destination is known at the time that the emergency service personnel are dispatched, the route from a starting point (e.g. hospital) to a destination point (e.g. accident location) can be traced on a digital map in an ESV equipped with a GPS unit. We call this route the ESV Route. The ESV periodically (with a pre-defined broadcast interval) broadcasts a report containing the following information:

- Unique ID of ESV (denoted by esv_id),

- Type of ESV (e.g. ambulance, police car or fire truck) (denoted by $\theta$ ),

- Start and end points (denoted by $\{s x, s y\}$ and $\{d x, d y\}$, respectively),

- $\quad$ Route Code (denoted $\Omega$ ),

- Tentative average speed of ESV on the ESV Route (denoted by $v_{e}$ ),

- Current ESV location coordinates and current time (denoted by $\{c x, c y\}$, and $t_{c}$ respectively),

- Timestamp denoting the time the report was sent by the ESV (denoted by 6 ).

Each ESV is assigned a unique ID, esv id, to distinguish reports sent by different ESVs within an area. The type of ESV, $\theta$, could help other motorists prepare for the kind of ESV for which they will have to clear the way. Start and end point coordinates along with the route information are vital in our approach. Each street is assigned a unique street ID. Vehicles are

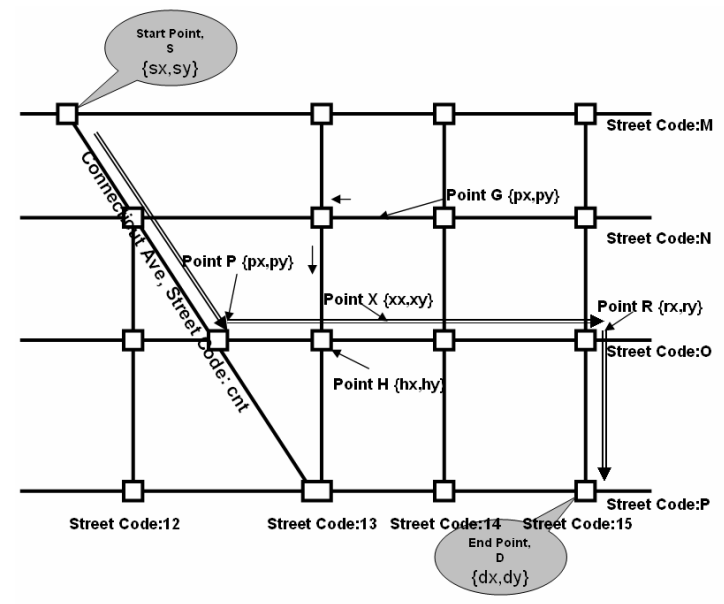

Figure 2. Illustration of the elements of an ESV route and the computation of time $t_{x}$. preloaded with digital maps that contain the street IDs for an entire city, or one or more states. The Route Code for an ESV Route is defined as a sequence of street codes with initial coordinates of each street. The street codes are ordered from the stating point to the end point. For example, in Figure 2, a solid line represents a street, and a small square at a crossing point of streets represents an intersection. S and D are the start and end points respectively for an ESV Route. The example route shown in Figure 2 can be traced in the following order: (1) S to P (i.e. Connecticut Ave., Street Code: cnt), (2) P to R (i.e. Street Code: O), and (3) R to D (i.e. Street Code: 15).

The starting point of each street on this ESV route can also be easily obtained: (1) Street Code: cnt - Point $\mathrm{S}\{\mathrm{sx}, \mathrm{sy}\},(2)$ Street Code: $\mathrm{O}-$ Point $\mathrm{P}\{\mathrm{px}, \mathrm{py}\}$, and (3) Street Code: 15 - Point R \{rx,ry\}. Therefore, the Route Code $\Omega=\{(s x, s y) / c n t, \quad(p x, p y) / O,(r x, r y) / 15\}$. The direction of the ESV route is determined by the order of streets.

Any vehicle that receives $\{s x, s y\},\{d x, d y\}$, and $\Omega$ from an ESV can reconstruct the actual ESV Route on its digital map by matching the street codes to $\Omega$. The tentative average speed of an ESV on a route $\Omega$ is a predicted average speed for the ESV. The current location coordinates and current time, $\{c x, c y\}$ and $t_{c}$, provide information about when and where the ESV was located when the report was sent. The timestamp along with the current location helps with sorting the reports for their relevance, as will be explained later. Each vehicle stores its current average speed, $v_{v}$, calculated during a past short period of time. The value of $v_{v}$ is periodically updated. Assume that at time $t_{g}$, a vehicle is located at point $\mathrm{G}$ on the street $\mathrm{N}$ (see Figure 2). We assume that at time $t_{x}$, the ESV and the vehicle will meet at point X on the ESV Route. With the ESV Route known to the vehicle, let $\mathrm{H}$ be the point where the vehicle will meet the ESV Route. Let $d_{v}$ be the distance on the vehicle's route between $\mathrm{G}$ and $\mathrm{H}$. Let $d_{e}$ be the distance on the ESV Route between $\{c x, c y\}$ and $H$. In Figure 2, point $X$ will be on the left side of $\mathrm{H}$ if the vehicle is moving in the opposite direction of the ESV Route. We call this Opposite Direction Movement (ODM). Similarly, point X will be on the right side of $\mathrm{H}$ if the vehicle is moving in the same direction as the ESV Route. We call this Same Direction Movement (SDM). Computation performed using the average ESV speed $\left(v_{e}\right)$, ESV location coordinates $(\{c x, c y\})$, and current time $\left(t_{c}\right)$ obtained from the report sent by an ESV yields: 
- $\quad t_{x}=\left(\left(d_{e}-d_{v}\right)+\left(v_{e}{ }^{*} t_{c}-v_{v}{ }^{*} t_{g}\right)\right) /\left(v_{e}-v_{v}\right)$ for SDM

- $t_{x}=\left(\left(d_{e}+d_{v}\right)+\left(v_{e}{ }^{*} t_{c}+v_{v}{ }^{*} t_{g}\right)\right) /\left(v_{e}+v_{v}\right)$ for ODM

With this computed $t_{x}$, a short period of clearing path time, $t_{c l r}(30 \mathrm{sec}$ or $1 \mathrm{~min}$ before the ESV meets the other vehicle), which is the time range during which the motorist should clear the way for the ESV can be provided to the motorist. Note that the value of $t_{x}$ is dynamic since $v_{e}$ and $v_{v}$ can both change.

\subsection{Selection strategy based on relevance}

Relevance describes the degree of applicability of a received report. The relevance of a resource report $R$ is calculated through a relevance function $r(R)=-\sigma-d_{e} / v_{e}$ [3]. During a vehicle's trip, it receives resource reports from resources or other vehicles. Periodically, reports in a vehicle's database are sorted according to their relevance. The most relevant report is used for computing $t_{x}$. For a vehicle, if a point $\mathrm{H}$ (see Figure 2) is forecast, i.e. the vehicle will travel on an ESV Route in the future, then $t_{x}$ is computed, otherwise the most relevant report is rebroadcast for other vehicles.

\subsection{Evacuation traffic chaos reduction approach}

Although a state's network of public radio stations can broadcast traffic and shelter information during evacuations, one of the main problems for motorists is filtering the information. An evacuee would only like to know what shelters are currently available near his or her current location and would likely not be interested in knowing the status of all shelters in a city. Real-time filtered information about the availability and location of shelters, gas stations, etc. can be utilized by the evacuating motorists. This would avoid the wastage of effort, time and fuel in searching for useful resources.

In our approach, parking places, gas stations, hospitals, schools, and shelters periodically broadcast reports that include the following information: (1) type of the resource, (2) availability of the resource, (3) location coordinates of the resource, and (4) timestamp denoting the time the report was sent. The current location along with the timestamp helps with sorting the reports. Note that $v_{e}$ is replaced by $v_{v}$ for stationary resources. These reports will be updated as resource availability changes. The information can be easily filtered with respect to a vehicle's position.

As illustrated in Figure 1, during an evacuation, the path of supply trucks traveling in the opposite direction of the evacuating contraflow traffic is severely impeded. We can employ our ESV Route Traffic

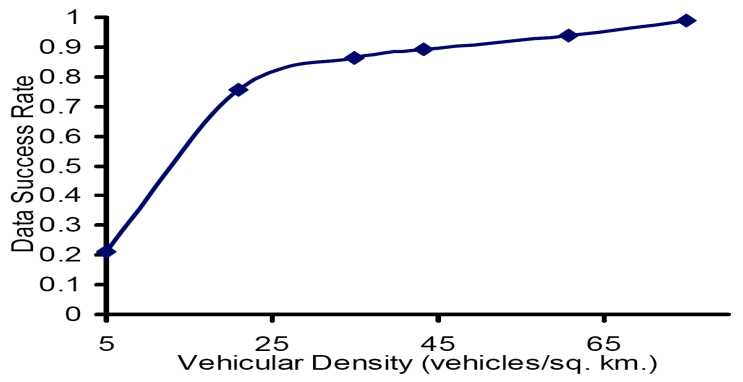

Figure 3. Data success rate vs. vehicular density.

Chaos Reduction Approach (described in Section 3.1) during an evacuation to clear the way for these needed supply trucks.

\section{Simulation results}

We consider a terrain size of $4000 \mathrm{~m} \times 4000 \mathrm{~m}$ (see Figure 2) with one source (S) and one destination (D). Nodes move in piecewise linear fashion, following city streets. Nodes move between $5-10 \mathrm{~m} / \mathrm{s}$ and ESVs move between $10-15 \mathrm{~m} / \mathrm{s}$. The broadcast interval is 10 seconds. The transmission range of vehicles is $300 \mathrm{~m}$. Figure 3 shows our preliminary results. Data success rate is defined as the ratio of the number of vehicles on the ESV route that have received the message within a certain response time to clear the path to the total number of vehicles on the ESV route. As expected, increasing the vehicular density will improve the success rate.

\section{Conclusions}

Based on our simulation results, we conclude that approaches to reduce traffic chaos in emergency scenarios are potentially attractive rollout applications for VANETs. Using GPS, digital map, and the selection strategies applied, we have achieved the goal of clearing the way for ESVs with minimal interruptions. Optimization of bandwidth consumption is left for future work.

\section{References}

[1] B. Wolshon and B. H. Meehan, "Emergency Evacuation: Ensuring Safe and Efficient Transportation Out of Endangered Areas", TR News, 224:3-9, Jan 2003.

[2] K. Li, "Evacuation Chaos, Death as Hurricane Surges On", The Age, Sept 25, 2005.

[3] O. Wolfson, B. Xu, and H. Yin, "Reducing Resource Discovery Time by Spatio-Temporal Information in Vehicular Ad-Hoc Networks", In Proceedings of ACM VANET, pages 91-92, Cologne, Germany, 2005. 